\title{
Epidermal Keratinocyte Sensing and Processing of Environmental Information Together with the Brain's Simulation and Prediction Abilities Helped to Enable Homo sapiens' Evolutionary Success
}

\author{
Mitsuhiro Denda ${ }^{1,2 *}$, Shinobu Nakanishi, ${ }^{1,2}$ \\ ${ }^{1}$ Japan Science and Technology Agency, CREST, Kawaguchi, Japan \\ ${ }^{2}$ Japan and Shiseido Global Innovation Center, Yokohama, Japan \\ Email: *mitsuhiro.denda@to.shiseido.co.jp
}

How to cite this paper: Denda, M., \& Nakanishi, S. (2020). Epidermal Keratinocyte Sensing and Processing of Environmental Information Together with the Brain's Simulation and Prediction Abilities Helped to Enable Homo sapiens Evolutionary Success. Advances in Anthropology, 10, 17-32. https://doi.org/10.4236/aa.2020.101002

Received: November 11, 2019

Accepted: December 13, 2019

Published: December 16, 2019

Copyright $\odot 2020$ by author(s) and Scientific Research Publishing Inc. This work is licensed under the Creative Commons Attribution International License (CC BY 4.0).

http://creativecommons.org/licenses/by/4.0/

\section{Open Access}

\begin{abstract}
Among terrestrial mammals, Homo sapiens has evolved a very specific anatomical feature-very little body hair-thus, the skin surface is exposed directly to the environment. We and others have demonstrated that skin epithelial cells, called keratinocytes, express not only functional sensory systems for a variety of environmental responses, but also a series of neurotransmitter receptors that play key roles in information processing in the brain. Furthermore, the brain cortex is particularly large in Homo sapiens, which has a higher ratio of brain to whole-body weight than any other mammalian species. Here we propose that the evolutionary success and global spread of $\mathrm{Ho}$ mo sapiens are due at least in part to the existence and interaction of these two systems; i.e. the epidermis and brain cortex. First, we discuss the role of the epidermis as a sophisticated organ with multiple sensory inputs and information-processing capabilities, and then we consider the putative requirement for a large brain to carry out simulations and predictions based on input from multiple epidermal systems. We also present some other examples where a functionally sophisticated epidermis is associated with a large brain size. Finally, we discuss possible reasons why Homo sapiens has emerged as the sole surviving human subspecies.
\end{abstract}

\section{Keywords}

Skin, Brain Cortex, Body Hair, Neurotransmitter 


\section{Introduction}

Surface coverings of living things have generally evolved to offer a survival advantage in their natural environment; for example, reptiles have protective scales, birds have feathers that are used to control flight, and terrestrial mammals have insulating body hair. But among terrestrial mammals, Homo sapiens evolved certain unique anatomical features-including loss of body hair-which exposed the skin surface directly to the environment, leaving only a water-impermeable membrane, the stratum corneum, at the outermost layer of the skin, as protection. Furthermore, another unusual anatomical characteristic of Homo sapiens is a large brain, especially the cerebral cortex section of the brain. Indeed, the ratio of brain to whole body weight; i.e. the encephalization quotient, is higher than in any other mammalian species. Among more than 200 primate species, only Homo sapiens possesses this combination of exposed skin and a large brain. Several questions then logically arise. What special advantage(s) do humans derive from their hairless skin? Could the combination of hairless skin and a large brain account for the evolutionary success of Homo sapiens? Then, did this unique combination contribute to the successful spread of Homo sapiens across the globe into an enormous range of environments, from hot, ultraviolet-B-enriched deserts to arctic regions?

In this article, we propose possible answers to these questions. Specifically, we hypothesize that the coevolution of a hairless epidermis with an enlarged brain (perhaps the latter "driven" by the former) provides the basis for the unique capabilities of Homo sapiens to control, manipulate and theorize about the human environment. We suggest that denuded epidermis receives and processes environmental information in real time. This input initiates emergency action when required, while also being passed on to the brain, where it is utilized for simulation/prediction to guide future action, which in turn could be modified by sensory feedback. The number of keratinocytes at the human body surface is estimated to be 100 billion. Thus, it is plausible that the epidermis generates huge amounts of information about its environment.

\section{Epidermal Keratinocytes as a Complex Sensory System}

While it has long been recognized that nerve terminals in the dermis (that extend non-myelinated nerve fibers into the overlying epidermis) play key roles in cutaneous sensation, in the past two decades, an enormous range of sensory functions has been discovered in epidermal keratinocytes (Table 1).

For example, we and others have shown that keratinocytes express a series of functional thermal receptors, transient receptor potential channels (TRPs) (Inoue et al., 2002, Tsutsumi et al., 2010a; Tsutsumi et al., 2011b; Denda, 2016). TRPV1 is activated by temperature $\left(>43^{\circ} \mathrm{C}\right)$, capsaicin, and acidic conditions, and activation of TRPV1 induces a sensation of pain (Caterina et al., 1997). Mice with keratinocyte-specific knockout of TRPV1 do not show pain-related avoidance responses after topical applications of irritants like capsaicin (Pang et al., 2015). 
Table 1. Physical and chemical factors sensed by keratinocytes.

\begin{tabular}{|c|c|c|c|}
\hline Environmental factors & Receptors & Role in sensing & References \\
\hline \multirow[t]{3}{*}{ Visible radiation } & $\mathrm{RHO}$ & Light and dark & Tsutsumi et al., 2009a \\
\hline & OPN1LW & Red-green light & Tsutsumi et al., 2009a \\
\hline & OPN1MW & Blue light & Tsutsumi et al., 2009a \\
\hline Electric potential & L-type VGCC & Electric potential & Denda et al., 2006 \\
\hline Magnetic field & Unknown & Magnetic energy & Lisi et al., 2006 \\
\hline High-frequency sound & Unknown & $10-30 \mathrm{kHz}$ sound & Denda \& Nakatani, 2010 \\
\hline \multirow[t]{10}{*}{ Temperature } & TRPV1 & Temperature $>43^{\circ} \mathrm{C}$ & Tsutsumi et al., 2011b \\
\hline & & Capsaicin, low $\mathrm{pH}<6.6$ & Inoue et al., 2002 \\
\hline & TRPV2 & Temperature $>52^{\circ} \mathrm{C}$ & Tsutsumi et al., 2011b \\
\hline & TRPV3 & Temperature $>33^{\circ} \mathrm{C}$ & Peier et al., 2002 \\
\hline & & Thymol, eugenol & Xu et al., 2006 \\
\hline & TRPV4 & Temperature $>34^{\circ} \mathrm{C}$ & Chung et al., 2003 \\
\hline & & Osmotic pressure & Liedtke, 2007 \\
\hline & TRPM8 & Temperature $<22^{\circ} \mathrm{C}$ & Tsutsumi et al., 2010a \\
\hline & & Menthol & Denda et al., 2010 \\
\hline & TRPA1 & Temperature $<17^{\circ} \mathrm{C}$ & Tsutsumi et al., 2010a \\
\hline Atmospheric pressure & Unknown & Changes of pressure & Ikeyama et al., 2013 \\
\hline Mechanical stress & Unknown & Touch & Tsutsumi et al., 2009b \\
\hline Oxygen & Unknown & Oxygen partial pressure & Boutin et al., 2008 \\
\hline Perfume & OR2AT4 & Sandalwood & Busse et al., 2014 \\
\hline
\end{tabular}

Mechanical stress also induces excitation of keratinocytes via elevation of intracellular calcium ion concentrations (Tsutsumi et al., 2009b). This information is transferred to the nervous system and recognized as tactile sensations (Tsutsumi et al., 2011a). Moehring et al. reported that ATP release, induced by mechanical stimulation of keratinocytes, is a critical component of baseline mammalian tactile sensation (Moehring et al., 2018). Osmotic pressure, atmospheric pressure, odorants, and changes in oxygen partial pressure also induce keratinocyte excitation (Denda \& Denda, 2007; Ikeyama et al., 2007; Boutin et al., 2008).

The effects of infrared and ultraviolet light on the epidermis are well known, and we showed further that visible light influences epidermal permeability barrier homeostasis (Denda \& Fuziwara, 2008). We also found that a series of photoreceptors expressed in the retina are also expressed in human epidermal keratinocytes (Tsutsumi et al., 2009a) (Figure 1).

We then demonstrated that both audible sounds and ultrasound influence epidermal permeability barrier homeostasis (Denda \& Nakatani, 2010). Sound at 5 $\mathrm{kHz}$ did not influence barrier recovery, whereas 10 (audible), 20 and 30 (ultrasound) $\mathrm{kHz}$ sound accelerated barrier recovery. Thus, epidermal keratinocytes 


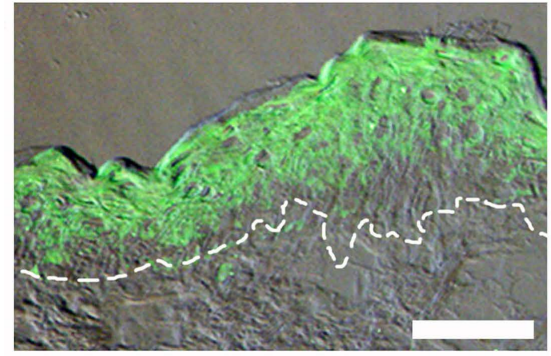

(a)

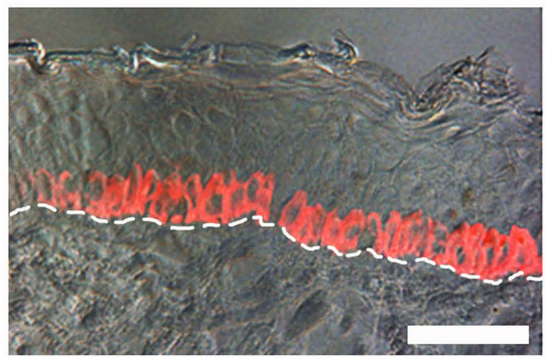

(c)

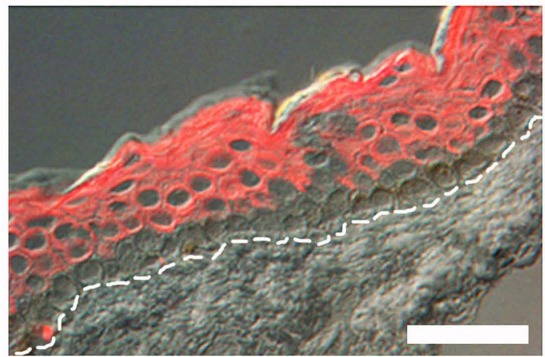

(e)

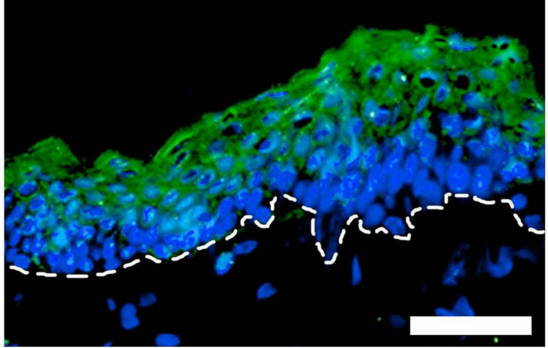

(b)

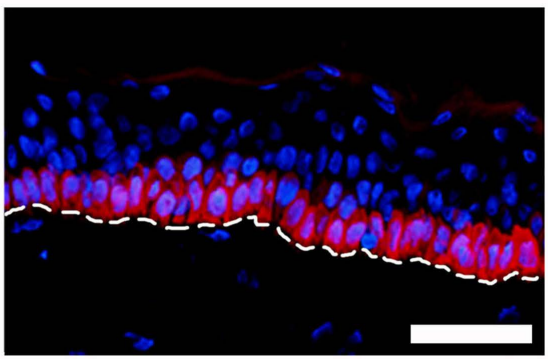

(d)

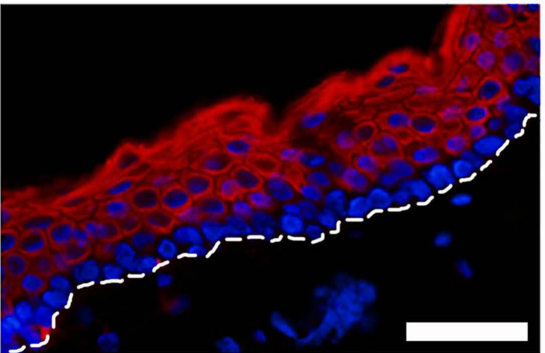

(f)

Figure 1. Photoreceptor expression in human epidermis. Human skin is studied immunohistochemically with the anti-rhodopsin (RHO) monoclonal antibody (RET-P1). Dotted lines indicate basal membrane at the epidermis/dermis. Marked immunoreactivity to RET-P1 was observed in the upper layer of the epidermis of human skin ((a) green color) Merged image with nuclear staining ((b) blue color). Immunohistochemical study of human skin with anti-Red Green-opsin (OPN1LW) ((c) red color) with nuclear staining ((d) blue color) and anti-Blue-opsin (OPN1MW) ((e) red color) with nuclear staining ((f) blue color). Marked immunoreactivity against anti-Red Green-opsin was observed at the basal layer of the epidermis of human skin, and immunoreactivity against anti-Blue-opsin was observed throughout the epidermis, except for the basal layer. It is possible a photoreceptor activated by longer-wavelength radiation (Red Green) would be localized in a deeper layer than receptors activated by shorter-wavelength radiation (Blue), because longer-wavelength radiation can penetrate deeper into the skin than shorter-wavelength radiation. Bar: $50 \mu \mathrm{m}$ (Modified from Tsutsumi et al., 2009a).

appear to sense a wider range of light and sound than either the eyes or ears, albeit with lower definition. Keratinocytes also express olfactory receptors (Busse et al., 2014), and a wide variety of chemicals can alter these receptors' electrochemical properties (Inoue at al., 2005, 2015). Furthermore, a variety of moleculesoriginally recognized as olfactory or gustatory molecules can improve skin pathology. For example, activation of olfactory receptor OR2AT4 accelerated wound healing (Busse et al., 2014), and topical application of fructose or man- 
nose accelerated the recovery of epidermal permeability barrier function after injury (Denda, 2011).

Other studies indicate that keratinocytes can sense magnetic fields (Lisi et al., 2006) and that epidermal homeostasis is influenced by the distribution of electrical potential (Denda \& Kumazawa, 2010; Denda et al., 2006). It is conceivable that keratinocytes might even sense cosmic radiation, because these particles can induce electromagnetic phenomena. It has also been suggested that quantum-mechanical phenomena, such as tunneling, might influence biochemical reactions (Cha et al., 1989). Thus, keratinocytes appear torespond to an enormous range of environmental phenomena.

\section{Epidermis as an Information-Processing System}

We have shown that a series of neurotransmitter receptors, which play key roles in information processing in the brain, are also expressed in keratinocytes (Denda et al., 2002a, 2002b, 2003a, 2003b, 2004; Fuziwara et al., 2003, 2005; Deing et al., 2013; Stojadinovic et al., 2013; Slominski et al., 2001; Wakamatsu et al., 1997). In addition, keratinocytes express a variety of neurohormones and receptors that are produced in the brain or induced by messages from the brain (Denda et al., 2012; Takei et al., 2013; Slominski et al., 2000). Hence, we speculate that the epidermis might possess information/emotion-processing capability, independent of the brain (Table 2).

For example, when different-shaped molds were pressed on the fingertip, different electrophysiological patterns were observed in peripheral nerves of the forearm, suggesting that geometrical information may be recognized by the skin (Pruszynski \& Johansson, 2014). Also, we have observed distinct spatiotemporal patterns of calcium concentration in monolayer-cultured human keratinocytes after physical or chemical stimulation (Tsutsumi et al., 2010b, 2013; Denda \& Tsutsumi, 2014). The

Table 2. Neurotransmitters, neuropeptides, and hormones generated by keratinocytes, and receptors functionally expressed in keratinocytes.

\begin{tabular}{ccc}
\hline Molecule & Receptor & References \\
\hline ATP & P2X, Y receptors & Inoue et al., 2005; Denda et al., 2002a \\
Glycine & Glycine receptor & Inoue et al., 2015 \\
GABA & GABA (A) receptor & Denda et al., 2002b \\
Adrenaline & Adrenergic $\beta 2$ receptor & Denda et al., 2003a \\
Acetylcholine & Cholinergic receptors & Denda et al., 2003b \\
Dopamine & Dopamine 2-like receptor & Fuziwara et al., 2005 \\
Glutamate & NMDA receptor & Fuziwara et al., 2003 \\
Oxytocin & Oxytocin receptor (OXTR) & Denda et al., 2012; Deing et al., 2013 \\
Cortisol & Glucocorticoid receptor (GR) & Stojadinovic et al., 2013 \\
CRF, ACTH & CRH receptors & Slominski et al., 2000, 2001 \\
& Melanocortin-1 receptor & Wakamatsu et al., 1997 \\
Nitric Oxide & & Ikeyama et al., 2007 \\
\hline
\end{tabular}


fingertip can recognize micron-scale patterns (Nakatani et al., 2011), but the density of peripheral nerve fibers in healthy skin is quite low (Tsutsumi et al., 2016) and may be inadequate to provide such fine discrimination. Therefore, it seems likely that epidermal keratinocytes are involved in information processing related to tactile sensation in the skin.

Individual neurons in the brain and keratinocytes show considerable similarities, in that they express comparable receptors and endocrinological systems, as described above, though their cell-cell communication systems are quite different. Neurons are connected via large numbers of synapses, forming a very complex network. On the other hand, cell-to-cell communication in the epidermis is less extensive (Tsutsumi et al., 2009b). A possible evolutionary explanation for this tiered system is to enable a very rapid response to environmental danger signals. Thus, when we encounter high temperatures or harmful chemicals, we move away from them quickly via spinal reflex (Giszter et al., 1989). On the other hand, some environmental signals might be sent to the brain as experiences that would be helpful to avoid future potential danger.

\section{The Brain as a System of Simulation and Prediction}

It has been shown that episodic memories are stored within the hippocampal-entorhinal cortex, while long-lasting memories are stored in the cerebral cortex (Kitamura et al., 2017). Thus, it seems feasible that the cerebral cortex might serve as a kind of virtual space in which stimuli from the external environment are processed and integrated based on information received from the sensory system, including the epidermis, and then used to formulate long-term strategies for coping with the world. This epidermal sensory group should be considered distinct from emergency response systems that interpret danger and would be consistent with the human capability for scientific theory-building. For example, though Yukawa predicted the existence of the meson as an elemental particle in 1935 (Yukawa, 1935), it was not detected until 1947 (Lattes et al., 1947). Similarly, Einstein predicted the effect of gravity on light in 1911 (Einstein, 1911), but the effect was only confirmed by astronomical observation by Eddington during an eclipse in 1919 (Eddington, 2011). How did Yukawa and Einstein predict such completely new phenomena? Wolfgang Pauli described the recognition of order in nature as follows: "The process of understanding, that is, in the conscious realization of new knowledge, seems thus to be based on a correspondence, a 'matching' of inner images pre-existent in the human psyche with external objects and their behavior" (Pauli, 2012). While this mechanism remains an open question from a current biological standpoint, simulation in the brain could be a mechanism to predict the existence of unknown phenomena outside of our bodily systems.

\section{Predictability of Biological Phenomena}

There is evidence that biological phenomena are susceptible to simulation and 
prediction. For example, we recently showed that computer simulation based on a mathematical model of epidermal homeostasis (an "in silico" epidermis) could be used to guide the development of a functionally capable epidermal equivalent system with a highly competent permeability barrier (Denda et al., 2014; Kobayashi et al., 2014, 2016; Kumamoto et al., 2018). Specifically, the simulations predicted that when the basal layer of the epidermal model felt undulations, the epidermis would become thicker and the stratum corneum would become flatter (Kobayashi \& Nagayama, 2016), and these predictions were verified in vitro (Kumamoto et al., 2018). Thus, it seems reasonable that our far more complex brain could utilize its enormous information input from the epidermis to predict the existence of such things as new elementary particles or quantum-mechanical effects. In other words, the information gathered by the epidermis might be used not only for responding rapidly to emergencies, but also for running long-term simulations in the "virtual space" of the cerebral cortex. Furthermore, this ability might have dramatically increased after humans invented writing for information storage and might still be increasing with the help of artificial intelligence (AI).

\section{Discussion}

\subsection{Speculation from the Standpoint of Comparative Anatomy}

We recently hypothesized a relationship between the shedding of hair from the skin of $H$. erectus and the evolution of larger brain size (Denda et al., 2018). We suggested that hairless skin offered an evolutionary advantage, because it was more efficient at sensing danger signals from the environment, because its sensory systems are more accessible. Then, because of the dramatically increased amount of environmental information generated from hairless skin, the brain size of hominins needed to expand in order to process all this sensory input efficiently. This hypothesis is supported by the finding that the plethora of peripheral sensations in the octopusis associated with a larger and more sentient brain. The octopus must integrate sensory inputs not only from its eight tentacles and its separate peripheral "brains", but also from its highly "sentient" skin. As a result, the octopus has a brain with 6-fold more neurons as compared to rodents (Hochner, 2012, 2013). Some species of squid have an even bigger brain; i.e. a higher ratio of brain/whole-body weight than the octopus (Packard, 1972). They also have the ability to change their body color pattern quickly for crypsis or communication (Hanlon et al., 1999). Furthermore, electric fish of the genus Gnathonemus possess a huge number of epidermal electroreceptors covering its whole body that allow these organisms to accurately assess the distance, size and shape of surrounding objects by transmitting an "electric image" to the brain (von der Emde et al., 1998). Indeed, the brain: body mass ratio of these fish is higher than that of any other vertebrate, including humans, and the brain accounts for $60 \%$ of bodily oxygen $\left(\mathrm{O}_{2}\right)$ consumption, compared to the $2 \%-8 \%$ in resting humans (Nilsson, 1996). These three examples suggest that the combina- 
tion of an exposed, environmentally sensitive skin and a large brain might have played a key role during animal evolution.

Several terrestrial mammals are largely hairless, including the hippopotamus, elephant, rhinoceros and naked mole rat, and the hippopotamus and rhinoceros do not have large brains (Lyras, 2018; Bhagwandin et al., 2017). We propose that because these two animals have thick skin, it might have been difficult to construct tightly-organized, arborizing peripheral nerve networks in their skin. Elephants have relatively large brains (Lyras, 2018), but their skin could also be too thick to construct peripheral nerve networks. We speculate instead that the elephant has a flexible arm, its long nose. To control this "arm", an elephant might need a bigger brain in comparison to a hippopotamus or rhinoceros. Consider the octopus which has eight flexible arms and substantial cognitive ability. Likewise, humans have two arms, each of which has ten flexible fingers. Flexible arms and fingers might require a larger brain. While naked mole rats admittedly do not have large brains (Kverková et al., 2018), they live at high population densities, making a complex sensory system unnecessary for their survival.

We can also look at this question in yet another way. A large group of arthropods-insects-have their entire bodies covered with a chitinous shell, with their tactile senses limited to their antennae. That is, they do not have skin sensations. Notably, their brain size is small, and the number of neurons in their brain is only around a million. Nevertheless, some of them, such as ants or bees, have complex social structures. Leaf-cutter ants carry out a kind of agriculture (Blanton \& Ewel, 1985) and honeybees can pass on very complex information (Menzel, 2012). Dragonflies can construct spatial and temporal images in their nervous systems to help catch their prey (Mischiati et al., 2015). Thus, even with a small brain, quite sophisticated actions are possible by discarding skin sensation, these insects can survive in a variety of environments without the high energy cost of a larger brain. The number of insect species is thought to be around one million, and they have persisted for over 400 million years, so this "protective shell-small brain-low energy" tactic has been successful and represents a valid alternative to the "exposed skin—big brain—high energy" strategy.

\subsection{Why Has Homo sapiens Outlasted Other Human Subspecies?}

Traces of early hominin subspecies, including Homo neanderthalensis and Denisova hominin, have been found only in limited areas of Eurasia. On the other hand, Homo sapiens reached Australia around 65,000 years ago and South America about 13,000 years ago at the latest. It has been suggested that because Homo sapiens have both "generalist" and "specialist" abilities (Roberts \& Stewart, 2018), they could successfully enter new ecological niches, and adapt to a variety of environments, allowing them to eventually colonize the entire world. Pertinently, the trait of autism (without intellectual impairment) is known to be associated with valuable technical and social skills (Spikins et al., 2016). Hence, autism might not be due to a lack of sociability, but rather to a lack of a rules-based theory of mind, and thus autism might be favorable for innovation 
and communication within certain cultural groups. Indeed, some genes implicated in autism are found in modern humans, but not in Homo neanderthalensis. Thus, autism might be required for an ability to be both a "generalist" and "specialist". Furthermore, because autism may render people less willing to be followers, Spikins et al. (2016) points out that people with autism often attain notable positions in society, particularly in the fields of medicine, engineering, mathematics, physics and information technology. Thus, a key point in human intellectual evolution might be tolerance of a variety of individual characteristics, including autism.

Green et al. (2010) sequenced the Homo neanderthalensis genome and identified ancestral genes that are "fixed" in present-day humans, together with others that changed over time in Homo sapiens, presumably due to positive selection (Green et al., 2010). Among the list of un-fixed genes of Homo neanderthalensis, several are related to epidermal keratinocytes. These include: 1) the RPTN gene encoding repetin, an epidermal structural protein and cornified cell envelope precursor that plays a crucial role in constructing the stratum corneum barrier (Huber et al., 2005); 2) the K1C16 gene encoding a keratin; 3) type I cytoskeletal keratin 16, which plays an important role in wound healing (Paladini et al., 1996); as well as 4) the OR2AT4 gene encoding olfactory receptor, family 2, subfamily AT, member 4, which accelerates wound healing (Busse et al., 2014). These results suggest that the epidermis and keratinocytes of Homo sapiens might be better protected from environmental insults than Homo neanderthalensis, and this capacity in turn may indicate their important role in a species' survival.

\section{Conclusion}

Homo sapiens is mostly unique among mammals in having a hairless epidermis, expressing a wide range of molecules that function in sensory and information-processing systems, as well as possessing the largest brain in terms of the ratio of brain to whole-body weight. Here, we present the hypothesis that the evolutionary success of Homo sapiens is at least in part due to the existence and cooperative action of these two systems. We suggest that keratinocytes that comprise the hairless epidermis are equipped with multiple sensory and information-processing systems that detect environmental information, both for immediate response to environmental danger signals, and for passage onward to the brain, where this information can be used for simulation and prediction to guide future actions, which in turn can be modified by further sensory feedback (Figure 2).

The "protective shell-small brain-low energy" strategy is robust, because huge numbers of species that employ it, such as insects, have survived over the past half billion years. On the other hand, among terrestrial mammals, only Homo sapiens has survived by employing the "exposed skin—big brain-high energy" strategy, while all other human subspecies have disappeared within the 


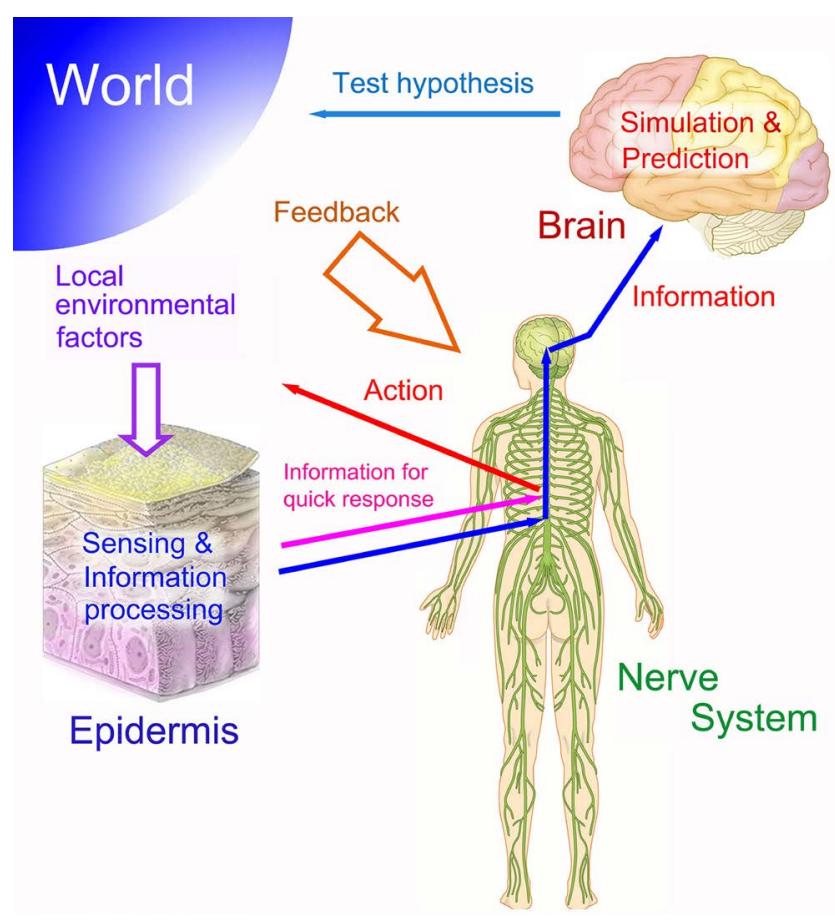

Figure 2. Schematic illustration of the putative roles of epidermal keratinocytes and brain. Epidermal keratinocytes sense a variety of environmental factors and stimuli. This information is processed in the epidermis and passed via the nervous system to the whole body and brain. In the brain, this information is used for simulations and predictions to guide future actions, which in turn may be modified by further sensory feedback.

past 30,000 years. These considerations suggest that the anatomical structure of Homo sapiens is very specific and potentially fragile in the face of mutations and evolutionary pressures.

\section{Acknowledgements}

This work was supported by JST CREST (Grant Number JPMJCR15D2). We appreciate constructive advice from Professor Peter M. Elias, University of California, San Francisco.

\section{Conflicts of Interest}

None declared.

\section{Author's Contributions}

MD wrote initial draft and provided initial concept. SN provided critical information about molecular biology aspects.

\section{Data Accessibility Statement}

Note: if data, scripts, or other arte facts used to generate the analyses presented in the paper are available via a publicly available data repository, authors should include a reference to the location of the material within their paper. 


\section{References}

Bhagwandin, A., Haagensen, M., \& Manger, P.R. (2017). The Brain of the Black (Diceros bicornis) and White (Ceratotherium simum) African Rhinoceroses: Morphology and Volumetrics from Magnetic Resonance Imaging. Frontiers in Neuroanatomy, 11, 74. https://doi.org/10.3389/fnana.2017.00074

Blanton, C. M., \& Ewel, J. J. (1985). Leaf-Cutting ant Herbivory in Successional and Agricultural Tropical Ecosystems. Ecology, 66, 861-869. https://doi.org/10.2307/1940548

Boutin, A. T., Weidemann, A. Z., Fu, L., Mesropian, K., Gradin, C., Jamora, M., Wiesener, K. U., Eckardt, C. J. et al. (2008). Epidermal Sensing of Oxygen Is Essential for Systemic Hypoxic Response. Cell, 133, 223-234. https://doi.org/10.1016/j.cell.2008.02.038

Busse, D., Kudella, P., Grüning, N. M., Gisselmann, G., Ständer, S., Luger, T. et al. (2014). A Synthetic Sandalwood Odorant Induces Wound-Healing Processes in Human Keratinocytes via the Olfactory Receptor OR2AT4. Journal of Investigative Dermatology, 134, 2823-2832. https://doi.org/10.1038/jid.2014.273

Caterina, M. J., Schumacher, M. A., Tominaga, M., Rosen, T. A., Levine, J. D., \& Julius, D. (1997). The Capsaicin Receptor: A Heat-Activated Ion Channel in the Pain Pathway. Nature, 389, 816-824. https://doi.org/10.1038/39807

Cha, Y., Murray, C. J., \& Klinman, J. P. (1989). Hydrogen Tunneling in Enzyme Reactions. Science, 243, 1325-1330. https://doi.org/10.1126/science.2646716

Chung, M. K., Lee, H., \& Caterina, M. J. (2003). Warm Temperatures Activate TRPV4 in Mouse 308 Keratinocytes. The Journal of Biological Chemistry, 278, 32037-32046. https://doi.org/10.1074/jbc.M303251200

Deing, V., Roggenkamp, D., Kühnl, J., Gruschka, A., Stäb, F., Wenck, H. et al. (2013). Oxytocin Modulates Proliferation and Stress Responses of Human Skin Cells: Implications for Atopic Dermatitis. Experimental Dermatology, 22, 399-405.

https://doi.org/10.1111/exd.12155

Denda, M. (2011). Effects of Topical Application of Aqueous Solutions of Hexoses on Epidermal Permeability Barrier Recovery Rate after Barrier Disruption. Experimental Dermatology, 20, 943-944. https://doi.org/10.1111/j.1600-0625.2011.01329.x

Denda, M. (2016). Sensing Environmental Factors: The Emerging Role of Receptors in Epidermal Homeostasis and Whole Body Health. In G. T. Wondrak (Ed.), Skin Stress Response Pathways: Environmental Factors and Molecular Opportunities (pp. 403-414). Cham: Springer. https://doi.org/10.1007/978-3-319-43157-4_19

Denda, M., \& Denda, S. (2007). Air-Exposed Keratinocytes Exhibited Intracellular Oscillation. Skin Research and Technology, 13, 195-201. https://doi.org/10.1111/j.1600-0846.2007.00210.x

Denda, M., \& Fuziwara, S. (2008). Visible Radiation Affects Epidermal Permeability Barrier Recovery: Selective Effects of Red and Blue Light. Journal of Investigative Dermatology, 128, 1335-1336. https://doi.org/10.1038/sj.jid.5701168

Denda, M., \& Kumazawa, N. (2010). Effects of Metals on Skin Permeability Barrier Recovery. Experimental Dermatology, 19, e124-e127. https://doi.org/10.1111/j.1600-0625.2009.01015.x

Denda, M., \& Nakatani, M. (2010). Acceleration of Permeability Barrier Recovery by Exposure of Skin to 10-30 Kilohertz Sound. British Journal of Dermatology, 162, 503-507. https://doi.org/10.1111/j.1365-2133.2009.09509.x

Denda, M., \& Tsutsumi, M. (2014). Possible Role of Epidermal Keratinocytes in the Construction of Acupuncture Meridians. Journal of Acupuncture and Meridian Studies, 7, 
92-94. https://doi.org/10.1016/j.jams.2013.03.002

Denda, M., Denda, S., Tsutsumi, M., Goto, M., Kumamoto, J., Nakatani, M. et al. (2014). Frontiers in Epidermal Barrier Homeostasis-An Approach to Mathematical Modeling of Epidermal Calcium Dynamics. Experimental Dermatology, 23, 79-82. https://doi.org/10.1111/exd.12302

Denda, M., Fuziwara, S., \& Hibino, T. (2006). Expression of Voltage-Gated Calcium Channel Subunit $\alpha \mathrm{C} 1$ in Epidermal Keratinocytes and Effects of Agonist and Antagonists of the Channel on Skin Barrier Homeostasis. Experimental Dermatology, 15, 455-460. https://doi.org/10.1111/j.0906-6705.2006.00430.x

Denda, M., Fuziwara, S., \& Inoue, K. (2003a). Beta-2-Adrenergic Receptor Antagonist Accelerates Skin Barrier Recovery and Reduces Epidermal Hyperplasia Induced by Barrier Disruption. Journal of Investigative Dermatology, 121, 142-148.

https://doi.org/10.1046/j.1523-1747.2003.12310.x

Denda, M., Fuziwara, S., \& Inoue, K. (2003b). Influx of Calcium and Chloride Ions into Epidermal Keratinocytes Regulates Exocytosis of Epidermal Lamellar Bodies and Skin Permeability Barrier Homeostasis. Journal of Investigative Dermatology, 121, 362-367. https://doi.org/10.1046/j.1523-1747.2003.12367.x

Denda, M., Fuziwara, S., Inoue, K. (2004). Association of Cyclic AMP with Permeability Barrier Homeostasis. Journal of Investigative Dermatology, 122, 140-146.

https://doi.org/10.1046/j.0022-202X.2003.22115.x

Denda, M., Inoue, K., Fuziwara, S., \& Denda, S. (2002a). P2X Purinergic Receptor Antagonist Accelerates Skin Barrier Repair and Prevents Epidermal Hyperplasia Induced by Skin Barrier Disruption. Journal of Investigative Dermatology, 119, 1034-1040. https://doi.org/10.1046/j.1523-1747.2002.19505.x

Denda, M., Inoue, K., Inomata, S., \& Denda, S. (2002b). GABA (A) Receptor Agonists Accelerate Cutaneous Barrier Recovery and Prevent Epidermal Hyperplasia Induced by Barrier Disruption. Journal of Investigative Dermatology, 119, 1041-1047. https://doi.org/10.1046/j.1523-1747.2002.19504.x

Denda, M., Menon, G. K., \& Elias, P. M. (2018). Did Hairlessness Stimulate an Increase in Hominin Brain Size? Insight from Cutaneous Neurosensory Interface and Comparative Vertebrate Morphology. Anthropology, 6, 1000199-1000199. https://doi.org/10.4172/2332-0915.1000199

Denda, M., Tsutsumi, M., \& Denda, S. (2010). Topical Application of TRPM8 Agonists Accelerates Skin Permeability Barrier Recovery and Reduces Epidermal Proliferation Induced by Barrier Insult: The Role of Cold-Sensitive TRP Receptors in Epidermal Permeability Barrier Homeostasis. Experimental Dermatology, 19, 791-795. https://doi.org/10.1111/j.1600-0625.2010.01154.x

Denda, S., Takei, K., Kumamoto, J., Goto, M., Tsutsumi, M., \& Denda, M. (2012). Oxytocin Is Expressed in Epidermal Keratinocytes and Released upon Stimulation with Adenosine 5'-[Gamma-Thio] Triphosphate in Vitro. Experimental Dermatology, 21, 535-537. https://doi.org/10.1111/j.1600-0625.2012.01507.x

Eddington, A. S. (2011). The Crucial Phenomena. In A. S. Eddington (Ed), Report on the Relativity Theory of Gravitation the Physical Society of London (pp. 48-58). London, UK: Forgotten Bools.

Einstein, A. (1911). Über den Einfluß der Schwerkraft auf die Ausbreitung des Lichtes. Annalen der Physik (Leipzig), 340, 898-908. https://doi.org/10.1002/andp.19113401005

Fuziwara, S., Inoue, K., \& Denda, M. (2003). NMDA-Type Glutamate Receptor Is Associated with Cutaneous Barrier Homeostasis. Journal of Investigative Dermatology, 120, 1023-1029. https://doi.org/10.1046/j.1523-1747.2003.12238.x 
Fuziwara, S., Suzuki, A., Inoue, K., \& Denda, M. (2005). Dopamine D2-Like Receptor Agonists Accelerate Barrier Repair and Inhibit the Epidermal Hyperplasia Induced by Barrier Disruption. Journal of Investigative Dermatology, 125, 783-789. https://doi.org/10.1111/j.0022-202X.2005.23873.x

Giszter, S. F., McIntyre, J., \& Bizzi, E. (1989). Kinematic Strategies and Sensorimotor Transformations in the Wiping Movements of Frogs. Journal of Neurophysiology, 62, 750-767. https://doi.org/10.1152/jn.1989.62.3.750

Green, R. E., Krause, J., Briggs, A. W., Maricic, T., Stenzel, U., Kircher, M. et al. (2010). A Draft Sequence of the Neandertal Genome. Science, 328, 710-722. https://doi.org/10.1126/science.1188021

Hanlon, R. T., Maxwell, M. R., Shashar N., Loew, E. R., \& Boyle, K. L. (1999). An ethogram of Body Patterning Behavior in the Biomedically and Commercially Valuable Squid Loligo pealei off Cape Cod, Massachusetts. The Biological Bulletin, 19, 49-62. https://doi.org/10.2307/1542996

Hochner, B. (2012). An Embodied View of Octopus Neurobiology. Current Biology, 22, R887-R892. https://doi.org/10.1016/j.cub.2012.09.001

Hochner, B. (2013). How Nervous Systems Evolve in Relation to Their Embodiment: What We Can Learn from Octopuses and Other Molluscs. Brain, Behavior and Evolution, 82, 19-30. https://doi.org/10.1159/000353419

Huber, M., Siegenthaler, G., Mirancea, N., Marenholz, I., Nizetic, D., Breitkreutz, D. et al. (2005). Isolation and Characterization of Human Repetin, a Member of the Fused Gene Family of the Epidermal Differentiation Complex. Journal of Investigative Dermatology, 124, 998-1007. https://doi.org/10.1111/j.0022-202X.2005.23675.x

Ikeyama, K., Fuziwara, S., \& Denda, M. (2007). Topical Application of Neuronal Nitric Oxide Synthase Inhibitor Accelerates Cutaneous Barrier Recovery and Prevents Epidermal Hyperplasia Induced by Barrier Disruption. Journal of Investigative Dermatology, 127, 1713-1719. https://doi.org/10.1038/sj.jid.5700742

Ikeyama, K., Nakatani, M., Kumamoto, J., \& Denda, M. (2013). Distinct Intracellular Calcium Responses of Individual Cultured Human Keratinocytes to Air Pressure Changes. Skin Research and Technology, 19, 346-351. https://doi.org/10.1111/srt.12045

Inoue, K., Denda, M., Tozaki, H., Fujishita, K., Koizumi, S., \& Inoue, K. (2005). Characterization of Multiple P2X Receptors in Cultured Normal Human Epidermal Keratinocytes. Journal of Investigative Dermatology, 124, 756-763.

https://doi.org/10.1111/j.0022-202X.2005.23683.x

Inoue, K., Koizumi, S., Fuziwara, S., Denda, S., Inoue, K., \& Denda, M. (2002). Functional Vanilloid Receptors in Cultured Normal Human Keratinocytes. Biochemical and Biophysical Research Communications, 291, 124-129. https://doi.org/10.1006/bbrc.2002.6393

Inoue, K., Takei, K., \& Denda, M. (2015). Functional Glycine Receptor in Cultured Human Keratinocytes. Experimental Dermatology, 24, 307-309. https://doi.org/10.1111/exd.12651

Kitamura, T., Ogawa, S. K., Roy, D. S., Okuyama, T., Morrissey, M. D. et al. (2017). Engrams and Circuits Crucial for Systems Consolidation of a Memory. Science, 356, 73-78. https://doi.org/10.1126/science.aam6808

Kobayashi, Y., \& Nagayama, M. (2016). Mathematical Model of Epidermal Structure. In R. S. Anderssen et al. (Eds.), Applications + Practical Conceptualization + Mathematics = Fruitful Innovation, Mathematics for Industry (Vol. 11, pp. 121-126). Tokyo, Japan: Springer. https://doi.org/10.1007/978-4-431-55342-7_11

Kobayashi, Y., Sanno, Y., Sakai, A., Sawabu, Y., Tustsumi, M., Goto, M. et al. (2014). Ma- 
thematical Modeling of Calcium Waves Induced by Mechanical Stimulation in Keratinocytes. PLOS ONE, 9, e92650. https://doi.org/10.1371/journal.pone.0092650

Kobayashi, Y., Sawabu, Y., Kitahata, H., Denda, M., \& Nagayama, M. (2016). Mathematical Model for Calcium-Assisted Epidermal Homeostasis. Journal of Theoretical Biology, 397, 52-60. https://doi.org/10.1016/j.jtbi.2016.02.032

Kumamoto, J., Nakanishi, S., Makita, M., Uesaka, M., Yasugahira, Y., Kobayashi, Y. et al. (2018). Mathematical-Model-Guided Development of Full-Thickness Epidermal Equivalent. Scientific Reports, 8, 17999. https://doi.org/10.1038/s41598-018-36647-y

Kverková, K., Bělíková, T., Olkowicz, S., Pavelková, Z., O’Riain, M. J., Šumbera, R., Burda, H., Bennett, N. C., \& Němec, P. (2018). Sociality Does Not Drive the Evolution of Large Brains in Eusocial African Mole-Rats. Scientific Reports, 8, 9203.

https://doi.org/10.1038/s41598-018-26062-8

Lattes, C. M. G., Muirhead, H., Occhialini, G. P. S., \& Powell, C. F. (1947). Processes Involving Charged Mesons. Nature, 159, 694-697. https://doi.org/10.1038/159694a0

Liedtke, W. (2007). Role of TRPV Ion Channels in Sensory Transduction of Osmotic Stimuli in Mammals. Experimental Physiology, 92, 507-512.

https://doi.org/10.1113/expphysiol.2006.035642

Lisi, A., Foletti, A., Ledda, M., Rosola, E., Giuliani, L., D’Emilia, E., \& Grimaldi, S. (2006). Extremely Low Frequency $7 \mathrm{~Hz} 100$ microT Electromagnetic Radiation Promotes Differentiation in the Human Epithelial Cell Line HaCaT. Electromagnetic Biology and Medicine, 25, 269-280. https://doi.org/10.1080/15368370601044184

Lyras, G. A. (2018). Brain Changes during Phyletic Dwarfing in Elephants and Hippos. Brain, Behavior and Evolution, 92, 167-181. https://doi.org/10.1159/000497268

Menzel, R. (2012). The Honeybee as a Model for Understanding the Basis of Cognition. Nature Reviews, 13, 758-768. https://doi.org/10.1038/nrn3357

Mischiati, M., Lin, H. T., Herold, P., Imler, E., Olberg, R., \& Leonardo, A. (2015). Internal Models Direct Dragonfly Interception Steering. Nature, 517, 333-338.

https://doi.org/10.1038/nature14045

Moehring, F., Cowie, A. M., Menzel, A. D., Weyer, A. D., Grzybowski, M., T., Arzua, T. et al. (2018). Keratinocytes Mediate Innocuous and Noxious Touch via ATP-P2X4 Signaling. eLife, 7, e31684. https://doi.org/10.7554/eLife.31684

Nakatani, M., Kawasoe, T., \& Denda, M. (2011). Sex Difference in Human Fingertip Recognition of Micron-Level Randomness as Unpleasant. International Journal of Cosmetic Science, 33, 346-350. https://doi.org/10.1111/j.1468-2494.2010.00634.x

Nilsson, G. (1996). Brain and Body Oxygen Requirements of Gnathonemus petersii, a Fish with an Exceptionally Large Brain. Journal of Experimental Biology, 199, 603-607.

Packard, A. (1972). Cephalopods and Fish: The Limit of Convergence. Biological Reviews, 47, 241-307. https://doi.org/10.1111/j.1469-185X.1972.tb00975.x

Paladini, R. D., Takahashi, K., Bravo, N. S., \& Coulombe, P. A. (1996). Onset of Re-Epithelialization after Skin Injury Correlates with a Reorganization of Keratin Filaments in Wound Edge Keratinocytes: Defining a Potential Role for Keratin 16. The Journal of Cell Biology, 132, 381-397. https://doi.org/10.1083/jcb.132.3.381

Pang, Z., Sakamoto, T., Tiwari, V., Kim, Y. S., Yang, F., Dong, X. et al. (2015). Selective Keratinocyte Stimulation Is Sufficient to Evoke Nociception in Mice. Pain, 156, 656-665. https://doi.org/10.1097/j.pain.0000000000000092

Pauli, W. (2012). The Influence of Archetypal Ideas on Kepler's Theories. In C. G. Jung, \& W. Pauli (Eds.), The Interpretation of Nature and the Psych (pp. 152). New York: Ishi Press. 
Peier, A. M., Reeve, A. J., Andersson, D. A., Moqrich, A., Earley, T. J., Hergarden A. C. et al. (2002). A Heat-Sensitive TRP Channel Expressed in Keratinocytes. Science, 296, 2046-2049. https://doi.org/10.1126/science.1073140

Pruszynski, J. A., \& Johansson, R. S. (2014). Edge-Orientation Processing in First-Order Tactile Neurons. Nature Neuroscience, 17, 1404-1409. https://doi.org/10.1038/nn.3804

Roberts, P., \& Stewart, B. A. (2018). Defining the 'Generalist Specialist' Niche for Pleistocene Homo sapiens. Nature Human Behaviour, 2, 542-550. https://doi.org/10.1038/s41562-018-0394-4

Slominski, A., Wortsman, J., Luger, T., Paus, R., \& Solomon, S. (2000). Corticotropin Releasing Hormone and Proopiomelanocortin Involvement in the Cutaneous Response to Stress. Physiological Reviews, 80, 979-1020. https://doi.org/10.1152/physrev.2000.80.3.979

Slominski, A., Wortsman, J., Pisarchik, A., Zbytek, B., Linton, E. A., Mazurkiewicz, J. E., \& Wei, E. T. (2001). Cutaneous Expression of Corticotropin-Releasing Hormone (CRH), Urocortin, and CRH Receptors. FASEB Journal, 15, 1678-1693. https://doi.org/10.1096/fj.00-0850rev

Spikins, P., Wright, B., \& Hodgson, D. (2016). Are There Alternative Adaptive Strategies to Human Pro-Sociality? The Role of Collaborative Morality in the Emergence of Personality Variation and Autistic Traits. Time \& Mind, 9, 289-313.

https://doi.org/10.1080/1751696X.2016.1244949

Stojadinovic, O., Sawaya, A., Pastar, I., \& Tomic-Canic, M. (2013). Glucocorticoid Receptor Localizes to Adherens Junctions at the Plasma Membrane of Keratinocytes. PLoS ONE, 30, e63453. https://doi.org/10.1371/journal.pone.0063453

Takei, K., Denda, S., Kumamoto, J., \& Denda, M. (2013). Low Environmental Humidity Induces Synthesis and Release of Cortisol in an Epidermal Organotypic Culture System. Experimental Dermatology, 22, 662-664. https://doi.org/10.1111/exd.12224

Tsutsumi, M., Denda, S., Ikeyama, K., Goto, M., \& Denda, M. (2010a). Exposure to Low Temperature Induces Elevation of Intracellular Calcium in Cultured Human Keratinocytes. Journal of Investigative Dermatology, 130, 1945-1948. https://doi.org/10.1038/jid.2010.33

Tsutsumi, M., Goto, M., \& Denda M. (2013). Dynamics of Intracellular Calcium in Cultured Human Keratinocytes after Localized Cell Damage. Experimental Dermatology, 22, 367-369. https://doi.org/10.1111/exd.12136

Tsutsumi, M., Goto, M., Denda, S., \& Denda, M. (2011a). Morphological and Functional Differences in Co-Culture System of Keratinocytes and Dorsal Root Ganglion-Derived Cells Depending on Time of Seeding. Experimental Dermatology, 20, 464-467.

https://doi.org/10.1111/j.1600-0625.2011.01276.x

Tsutsumi, M., Ikeyama, K., Denda, S., Nakanishi, J., Fuziwara, S., Aoki, H., \& Denda, M. (2009a). Expressions of Rod and Cone Photoreceptor-Like Proteins in Human Epidermis. Experimental Dermatology, 18, 567-570. https://doi.org/10.1111/j.1600-0625.2009.00851.x

Tsutsumi, M., Kitahata, H., Fukuda, M., Kumamoto, J., Goto, M., Denda, S. et al. (2016). Numerical and Comparative Three-Dimensional Structural Analysis of Peripheral Nerve Fibers in Epidermis of Atopic Dermatitis Patients. British Journal of Dermatology, 174, 191-194. https://doi.org/10.1111/bjd.13974

Tsutsumi, M., Kitahata, H., Nakata, S., Sanno, Y., Nagayama, M., \& Denda, M. (2010b). Mathematical Analysis of Intercellular Calcium Propagation Induced by ATP. Skin Research and Technology, 16, 146-150.

https://doi.org/10.1111/j.1600-0846.2009.00420.x 
Tsutsumi, M., Kumamoto, J., \& Denda, M. (2011b). Intracellular Calcium Response to High Temperatureis Similar in Undifferentiated and Differentiated Cultured Human Keratinocytes. Experimental Dermatology, 20, 839-840.

https://doi.org/10.1111/j.1600-0625.2011.01318.x

Tsutusmi, M., Inoue, K., Denda, S., Ikeyama, K., Goto, M., \& Denda, M. (2009b). Mechanical-Stimulation-Evoked Calcium Waves in Proliferating and Differentiated $\mathrm{Hu}-$ man Keratinocytes. Cell and Tissue Research, 338, 99-106.

https://doi.org/10.1007/s00441-009-0848-0

von der Emde, G., Schwarz, S., Gomez, L., Budelli, R., \& Grant K. (1998). Electric Fish Measure Distance in the Dark. Nature, 395, 890-894. https://doi.org/10.1038/27655

Wakamatsu, K., Graham, A., Cook, D., \& Thody, A. J. (1997). Characterization of ACTH Peptides in Human Skin and Their Activation of the Melanocortin-1 Receptor. Pigment Cell Research, 10, 288-297. https://doi.org/10.1111/j.1600-0749.1997.tb00688.x

Xu, H., Delling, M., Jun, J. C., \& Clapham, D. E. (2006). Oregano, Thyme and Clove-Derived Flavors and Skin Sensitizers Activate Specific TRP Channels. Nature Neuroscience, 9, 628-635. https://doi.org/10.1038/nn1692

Yukawa, H. (1935). On the Interaction of Elementary Particles. I. Proceedings of the Physico-Mathematical Society of Japan, 17, 48-57. 